\title{
Integral Cayley graphs defined by greatest common divisors
}

\author{
Walter Klotz \\ Institut für Mathematik \\ Technische Universität Clausthal, Germany \\ klotz@math.tu-clausthal.de \\ Torsten Sander \\ Fakultät für Informatik \\ Ostfalia Hochschule für angewandte Wissenschaften, Germany \\ t.sander@ostfalia.de
}

Submitted: Dec 6, 2010; Accepted: Apr 12, 2011; Published: Apr 21, 2011

Mathematics Subject Classification: 05C25, 05C50

\begin{abstract}
An undirected graph is called integral, if all of its eigenvalues are integers. Let $\Gamma=Z_{m_{1}} \otimes \cdots \otimes Z_{m_{r}}$ be an abelian group represented as the direct product of cyclic groups $Z_{m_{i}}$ of order $m_{i}$ such that all greatest common divisors $\operatorname{gcd}\left(m_{i}, m_{j}\right) \leq 2$ for $i \neq j$. We prove that a Cayley graph $\operatorname{Cay}(\Gamma, S)$ over $\Gamma$ is integral, if and only if $S \subseteq \Gamma$ belongs to the the Boolean algebra $B(\Gamma)$ generated by the subgroups of $\Gamma$. It is also shown that every $S \in B(\Gamma)$ can be characterized by greatest common divisors.
\end{abstract}

\section{Introduction}

The greatest common divisor of nonnegative integers $a$ and $b$ is denoted by $\operatorname{gcd}(a, b)$. Let us agree upon $\operatorname{gcd}(0, b)=b$. If $x=\left(x_{1}, \ldots, x_{r}\right)$ and $m=\left(m_{1}, \ldots, m_{r}\right)$ are tuples of nonnegative integers, then we set

$$
\operatorname{gcd}(x, m)=\left(d_{1}, \ldots, d_{r}\right)=d, \quad d_{i}=\operatorname{gcd}\left(x_{i}, m_{i}\right) \text { for } i=1, \ldots, r .
$$

For an integer $n \geq 1$ we denote by $Z_{n}$ the additive group, respectively the ring of integers modulo $n, Z_{n}=\{0,1, \ldots, n-1\}$ as a set. Let $\Gamma$ be an (additive) abelian group represented as a direct product of cyclic groups.

$$
\Gamma=Z_{m_{1}} \otimes \cdots \otimes Z_{m_{r}}, m_{i} \geq 1 \text { for } i=1, \ldots, r
$$


Suppose that $d_{i}$ is a divisor of $m_{i}, 1 \leq d_{i} \leq m_{i}$, for $i=1, \ldots, r$. For the divisor tuple $d=\left(d_{1}, \ldots, d_{r}\right)$ of $m=\left(m_{1}, \ldots, m_{r}\right)$ we define the $g c d$-set of $\Gamma$ with respect to $d$,

$$
S_{\Gamma}(d)=\left\{x=\left(x_{1}, \ldots, x_{r}\right) \in \Gamma: \operatorname{gcd}(x, m)=d\right\} .
$$

If $D=\left\{d^{(1)}, \ldots, d^{(k)}\right\}$ is a set of divisor tuples of $m$, then the ged-set of $\Gamma$ with respect to $D$ is

$$
S_{\Gamma}(D)=\bigcup_{j=1}^{k} S_{\Gamma}\left(d^{(j)}\right) .
$$

In Section 2 we realize that the gcd-sets of $\Gamma$ constitute a Boolean subalgebra $B_{g c d}(\Gamma)$ of the Boolean algebra $B(\Gamma)$ generated by the subgroups of $\Gamma$. The finite abelian group $\Gamma$ is called a gcd-group, if $B_{\text {gcd }}(\Gamma)=B(\Gamma)$. We show that $\Gamma$ is a gcd-group, if and only if it is cyclic or isomorphic to a group of the form

$$
Z_{2} \otimes \cdots \otimes Z_{2} \otimes Z_{n}, n \geq 2
$$

Eigenvalues of an undirected graph $G$ are the eigenvalues of an arbitrary adjacency matrix of $G$. Harary and Schwenk [8] defined $G$ to be integral, if all of its eigenvalues are integers. For a survey of integral graphs see [3]. In [2] the number of integral graphs on $n$ vertices is estimated. Known characterizations of integral graphs are restricted to certain graph classes, see e.g. [1]. Here we concentrate on integral Cayley graphs over ged-groups.

Let $\Gamma$ be a finite, additive group, $S \subseteq \Gamma, 0 \notin S,-S=\{-s: s \in S\}=S$. The undirected Cayley graph over $\Gamma$ with shift set $S, \operatorname{Cay}(\Gamma, S)$, has vertex set $\Gamma$. Vertices $a, b \in \Gamma$ are adjacent, if and only if $a-b \in S$. For general properties of Cayley graphs we refer to Godsil and Royle [7] or Biggs [5]. We define a gcd-graph to be a Cayley graph $\operatorname{Cay}(\Gamma, S)$ over an abelian group $\Gamma=Z_{m_{1}} \otimes \cdots \otimes Z_{m_{r}}$ with a gcd-set $S$ of $\Gamma$. All gcd-graphs are shown to be integral. They can be seen as a generalization of unitary Cayley graphs and of circulant graphs, which have some remarkable properties and applications (see [4], [9], [11], [15]).

In our paper [10] we proved for an abelian group $\Gamma$ and $S \in B(\Gamma), 0 \notin S$, that the Cayley graph $\operatorname{Cay}(\Gamma, S)$ is integral. We conjecture the converse to be true for finite abelian groups in general. This can be confirmed for cyclic groups by a theorem of So [16]. In Section 3 we extend the result of So to gcd-groups. A Cayley graph $\operatorname{Cay}(\Gamma, S)$ over a gcd-group $\Gamma$ is integral, if and only if $S \in B(\Gamma)$.

\section{2 gcd-Groups}

Throughout this section $\Gamma$ denotes a finite abelian group given as a direct product of cyclic groups,

$$
\Gamma=Z_{m_{1}} \otimes \cdots \otimes Z_{m_{r}}, m_{i} \geq 1 \text { for } i=1, \ldots, r .
$$

Theorem 1. The family $B_{g c d}(\Gamma)$ of gcd-sets of $\Gamma$ constitutes a Boolean subalgebra of the Boolean algebra $B(\Gamma)$ generated by the subgroups of $\Gamma$. 
Proof. First we confirm that $B_{g c d}(\Gamma)$ is a Boolean algebra with respect to the usual set operations. From $S_{\Gamma}(\emptyset)=\emptyset$ we know $\emptyset \in B_{g c d}(\Gamma)$. If $D_{0}$ denotes the set of all (positive) divisor tuples of $m=\left(m_{1}, \ldots, m_{r}\right)$ then we have $S_{\Gamma}\left(D_{0}\right)=\Gamma$, which implies $\Gamma \in B_{g c d}(\Gamma)$. As $B_{g c d}(\Gamma)$ is obviously closed under the set operations union, intersection and forming the complement, it is a Boolean algebra.

In order to show $B_{g c d}(\Gamma) \subseteq B(\Gamma)$, it is sufficient to prove for an arbitrary divisor tuple $d=\left(d_{1}, \ldots, d_{r}\right)$ of $m=\left(m_{1}, \ldots, m_{r}\right)$ that

$$
S_{\Gamma}(d)=\left\{x=\left(x_{1}, \ldots, x_{r}\right) \in \Gamma: \operatorname{gcd}(x, m)=d\right\} \in B(\Gamma) .
$$

Observe that $d_{j}=m_{j}$ forces $x_{j}=0$ for $x=\left(x_{i}\right) \in S_{\Gamma}(d)$. If $d_{i}=m_{i}$ for every $i=1, \ldots, r$ then $S_{\Gamma}(d)=\{(0,0, \ldots, 0)\} \in B(\Gamma)$. So we may assume $1 \leq d_{i}<m_{i}$ for at least one $i \in\{1, \ldots, r\}$. For $i=1, \ldots, r$ we define $\delta_{i}=d_{i}$, if $d_{i}<m_{i}$, and $\delta_{i}=0$, if $d_{i}=m_{i}$, $\delta=\left(\delta_{1}, \ldots, \delta_{r}\right)$. For $a_{i} \in Z_{m_{i}}$ we denote by $\left[a_{i}\right]$ the cyclic group generated by $a_{i}$ in $Z_{m_{i}}$. One can easily verify the following representation of $S_{\Gamma}(d)$ :

$$
S_{\Gamma}(d)=\left[\delta_{1}\right] \otimes \cdots \otimes\left[\delta_{r}\right] \backslash \bigcup_{\lambda_{1}, \ldots, \lambda_{r}}\left(\left[\lambda_{1} \delta_{1}\right] \otimes \cdots \otimes\left[\lambda_{r} \delta_{r}\right]\right) .
$$

In (1) we set $\lambda_{i}=0$, if $\delta_{i}=0$. For $i \in\{1, \ldots, r\}$ and $\delta_{i}>0$ the range of $\lambda_{i}$ is

$$
1 \leq \lambda_{i}<\frac{m_{i}}{\delta_{i}} \text { such that } \operatorname{gcd}\left(\lambda_{i}, \frac{m_{i}}{\delta_{i}}\right)>1 \text { for at least one } i \in\{1, \ldots, r\} \text {. }
$$

As $\left[\delta_{1}\right] \otimes \cdots \otimes\left[\delta_{r}\right]$ and $\left[\lambda_{1} \delta_{1}\right] \otimes \cdots \otimes\left[\lambda_{r} \delta_{r}\right]$ are subgroups of $\Gamma,(1)$ implies $S_{\Gamma}(d) \in B(\Gamma)$.

A gcd-graph is a Cayley graph $\operatorname{Cay}\left(\Gamma, S_{\Gamma}(D)\right)$ over an abelian group $\Gamma=Z_{m_{1}} \otimes \cdots \otimes$ $Z_{m_{r}}$ with a gcd-set $S_{\Gamma}(D)$ as its shift set. In [10] we proved that for a finite abelian group $\Gamma$ and $S \in B(\Gamma), 0 \notin S$, the Cayley graph $\operatorname{Cay}(\Gamma, S)$ is integral. Therefore, Theorem 1 implies the following corollary.

Corollary 1. Every gcd-graph $\operatorname{Cay}\left(\Gamma, S_{\Gamma}(D)\right)$ is integral.

We remind that we call $\Gamma$ a gcd-group, if $B_{g c d}(\Gamma)=B(\Gamma)$. For $a=\left(a_{i}\right) \in \Gamma$ we denote by $[a]$ the cyclic subgroup of $\Gamma$ generated by $a$.

Lemma 1. Let $\Gamma$ be the abelian group $Z_{m_{1}} \otimes \cdots \otimes Z_{m_{r}}, m=\left(m_{1}, \ldots, m_{r}\right)$. Then $\Gamma$ is a gcd-group, if and only if for every $a \in \Gamma, \operatorname{gcd}(a, m)=d$ implies $S_{\Gamma}(d) \subseteq[a]$.

Proof. Let $\Gamma$ be a gcd-group, $B_{\text {gcd }}(\Gamma)=B(\Gamma)$. Then every subgroup of $\Gamma$, especially every cyclic subgroup $[a]$ is a gcd-set of $\Gamma$. This means $[a]=S_{\Gamma}(D)$ for a set $D$ of divisor tuples of $m$. Now $\operatorname{gcd}(a, m)=d$ implies $d \in D$ and therefore $S_{\Gamma}(d) \subseteq S_{\Gamma}(D)=[a]$.

To prove the converse assume that the condition in Lemma 1 is satisfied. Let $H$ be an arbitrary subgroup of $\Gamma$. We show $H \in B_{g c d}(\Gamma)$. Let $a \in H, \operatorname{gcd}(a, m)=d$. Then our assumption implies

$$
a \in S_{\Gamma}(d) \subseteq[a] \subseteq H, \quad H=\bigcup_{d \in D} S_{\Gamma}(d)=S_{\Gamma}(D) \in B_{g c d}(\Gamma),
$$

where $D=\{\operatorname{gcd}(a, m): a \in H\}$. 
For integers $x, y, n$ we express by $x \equiv y \bmod n$ that $x$ is congruent to $y$ modulo $n$.

Lemma 2. Every cyclic group $\Gamma=Z_{n}, n \geq 1$, is a gcd-group.

Proof. As the lemma is trivially true for $n=1$, we assume $n \geq 2$. Let $a \in \Gamma, 0 \leq a \leq n-1$, $\operatorname{gcd}(a, n)=d$. According to Lemma 1 we have to show $S_{\Gamma}(d) \subseteq[a]$. Again, to avoid the trivial case, assume $a \geq 1$. From $\operatorname{gcd}(a, n)=d<n$ we deduce

$$
a=\alpha d, 1 \leq \alpha<\frac{n}{d}, \operatorname{gcd}\left(\alpha, \frac{n}{d}\right)=1 .
$$

As the order of $a \in \Gamma$ is $\operatorname{ord}(a)=n / d$, the cyclic group generated by $a$ is

$$
[a]=\left\{x \in \Gamma: x \equiv(\lambda \alpha) d \bmod n, 0 \leq \lambda<\frac{n}{d}\right\} .
$$

Finally, we conclude

$$
\begin{aligned}
{[a] } & \supseteq\left\{x \in \Gamma: x \equiv(\lambda \alpha) d \quad \bmod n, 0 \leq \lambda<\frac{n}{d}, \operatorname{gcd}\left(\lambda, \frac{n}{d}\right)=1\right\} \\
& =\left\{x \in \Gamma: x \equiv \mu d \quad \bmod n, 0 \leq \mu<\frac{n}{d}, \operatorname{gcd}\left(\mu, \frac{n}{d}\right)=1\right\}=S_{\Gamma}(d) .
\end{aligned}
$$

Lemma 3. If $\Gamma=Z_{m_{1}} \otimes \cdots \otimes Z_{m_{r}}, r \geq 2$, is a gcd-group, then $\operatorname{gcd}\left(m_{i}, m_{j}\right) \leq 2$ for every $i \neq j, i, j=1, \ldots, r$.

Proof. Without loss of generality we concentrate on $\operatorname{gcd}\left(m_{1}, m_{2}\right)$. We may assume $m_{1}>2$ and $m_{2}>2$. Consider $a=(1,1,0, \ldots, 0) \in \Gamma$ and $b=\left(m_{1}-1,1,0, \ldots, 0\right) \in \Gamma$. For $m=\left(m_{1}, \ldots, m_{r}\right)$ we have

$$
\operatorname{gcd}(a, m)=\left(1,1, m_{3}, \ldots, m_{r}\right)=\operatorname{gcd}(b, m) .
$$

By Lemma 1 the element $b$ must belong to the cyclic group $[a]$. This requires the existence of an integer $\lambda, b=\lambda a$ in $\Gamma$, or equivalently

$$
\lambda \equiv-1 \quad \bmod m_{1} \text { and } \lambda \equiv 1 \bmod m_{2} .
$$

Therefore, integers $k_{1}$ and $k_{2}$ exist satisfying $\lambda=-1+k_{1} m_{1}$ and $\lambda=1+k_{2} m_{2}$, which implies $k_{1} m_{1}-k_{2} m_{2}=2$ and $\operatorname{gcd}\left(m_{1}, m_{2}\right)$ divides 2 .

The next two lemmas will enable us to prove the converse of Lemma 3 .

Lemma 4. Let $a_{1}, \ldots, a_{r}, g_{1}, \ldots, g_{r}$ be integers, $r \geq 2, g_{i} \geq 2$ for $i=1, \ldots, r$. Moreover, assume $\operatorname{gcd}\left(g_{i}, g_{j}\right)=2$ for every $i \neq j, i, j=1, \ldots, r$. The system of congruences

$$
x \equiv a_{1} \bmod g_{1}, \ldots, x \equiv a_{r} \bmod g_{r}
$$

is solvable, if and only if

$$
a_{i} \equiv a_{j} \quad \bmod 2 \text { for every } i, j=1, \ldots, r .
$$

If the system is solvable, then the solution consists of a unique residue class modulo $\left(g_{1} g_{2} \cdots g_{r}\right) / 2^{r-1}$. 
Proof. Suppose that $x$ is a solution of (2). As every $g_{i}$ is even, the necessity of condition (3) follows by

$$
a_{i} \equiv x \quad \bmod 2 \text { for } i=1, \ldots, r .
$$

Assume now that condition (3) is satisfied. We set $\kappa=0$, if every $a_{i}$ is even, and $\kappa=1$, if every $a_{i}$ is odd. By $x \equiv a_{i} \bmod 2$ we have $x=2 y+\kappa$ for an integer $y$. The congruences $(2)$ can be equivalently transformed to

$$
y \equiv \frac{a_{1}-\kappa}{2} \quad \bmod \frac{g_{1}}{2}, \ldots, y \equiv \frac{a_{r}-\kappa}{2} \quad \bmod \frac{g_{r}}{2} .
$$

As $\operatorname{gcd}\left(\left(g_{i} / 2\right),\left(g_{j} / 2\right)\right)=1$ for $i \neq j, i, j=1, \ldots, r$, we know by the Chinese remainder theorem [14] that the system (4) has a unique solution $y \equiv h \bmod \left(g_{1} \cdots g_{r}\right) / 2^{r}$. This implies for the solution $x$ of (2):

$$
x=2 y+\kappa \equiv 2 h+\kappa \quad \bmod \quad \frac{g_{1} \cdots g_{r}}{2^{r-1}} .
$$

Lemma 5. Let $a_{1}, \ldots, a_{r}, m_{1}, \ldots, m_{r}$ be integers, $r \geq 2, m_{i} \geq 2$ for $i=1, \ldots, r$. Moreover, assume $\operatorname{gcd}\left(m_{i}, m_{j}\right) \leq 2$ for every $i \neq j, i, j=1, \ldots, r$. The system of congruences

$$
x \equiv a_{1} \bmod m_{1}, \ldots, x \equiv a_{r} \bmod m_{r}
$$

is solvable, if and only if

$$
a_{i} \equiv a_{j} \quad \bmod 2 \text { for every } i \neq j, m_{i} \equiv m_{j} \equiv 0 \quad \bmod 2, i, j=1, \ldots, r .
$$

Proof. If at most one of the integers $m_{i}, i=1, \ldots r$, is even then $\operatorname{gcd}\left(m_{i}, m_{j}\right)=1$ for every $i \neq j, i, j=1, \ldots, r$, and system (5) is solvable. Therefore, we may assume that $m_{1}, \ldots, m_{k}$ are even, $2 \leq k \leq r$, and $m_{k+1}, \ldots, m_{r}$ are odd, if $k<r$. Now we split system (5) into two systems.

$$
\begin{gathered}
x \equiv a_{1} \bmod m_{1}, \ldots, x \equiv a_{k} \bmod m_{k} \\
x \equiv a_{k+1} \bmod m_{k+1}, \ldots, x \equiv a_{r} \bmod m_{r}
\end{gathered}
$$

By Lemma 4 the solvability of (7) requires (6). If this condition is satisfied, then (7) has a unique solution $x \equiv b \bmod \left(m_{1} \cdots m_{k}\right) / 2^{k-1}$ by Lemma 4 . System (8) has a unique solution $x \equiv c$ mod $\left(m_{k+1} \cdots m_{r}\right)$ by the Chinese remainder theorem, because $\operatorname{gcd}\left(m_{i}, m_{j}\right)=1$ for $i \neq j, i, j=k+1, \ldots, r$. So the original system (5) is equivalent to

$$
x \equiv b \quad \bmod \frac{m_{1} \cdots m_{k}}{2^{k-1}} \text { and } x \equiv c \quad \bmod \left(m_{k+1} \cdots m_{r}\right) \text {. }
$$

As $\operatorname{gcd}\left(\left(m_{1} \cdots m_{k}\right),\left(m_{k+1} \cdots m_{r}\right)\right)=1$, the Chinese remainder theorem can be applied once more to arrive at a unique solution $x \equiv h \bmod \left(m_{1} \cdots m_{r}\right) / 2^{k-1}$ of $(9)$ and (5). 
Theorem 2. The abelian group $\Gamma=Z_{m_{1}} \otimes \cdots \otimes Z_{m_{r}}$ is a gcd-group, if and only if

$$
\operatorname{gcd}\left(m_{i}, m_{j}\right) \leq 2 \text { for every } i \neq j, i, j=1, \ldots, r .
$$

Proof. As every cyclic group is a gcd-group by Lemma 2, we may assume $r \geq 2$. Then (10) necessarily holds for every gcd-group $\Gamma$ by Lemma 3.

Suppose now that $\Gamma$ satisfies (10). Let $a=\left(a_{1}, \ldots, a_{r}\right)$ and $b=\left(b_{1}, \ldots, b_{r}\right)$ be elements of $\Gamma, m=\left(m_{1}, \ldots, m_{r}\right)$, and

$$
\operatorname{gcd}(a, m)=d=\left(d_{1}, \ldots, d_{r}\right)=\operatorname{gcd}(b, m) .
$$

According to Lemma 1 we have to show that $b$ belongs to the cyclic group $[a]$ generated by $a$. Now $b \in[a]$ is equivalent to the existence of an integer $\lambda$ which solves the following system of congruences:

$$
b_{1} \equiv \lambda a_{1} \quad \bmod m_{1}, \ldots, b_{r} \equiv \lambda a_{r} \quad \bmod m_{r} .
$$

If $d_{i}=m_{i}$ then $a_{i}=b_{i}=0$ and the congruence $b_{i} \equiv \lambda a_{i} \quad \bmod m_{i}$ becomes trivial. Therefore, we assume $1 \leq d_{i}<m_{i}$ for every $i=1, \ldots, r$. By (11) we have $\operatorname{gcd}\left(a_{i}, m_{i}\right)=$ $\operatorname{gcd}\left(b_{i}, m_{i}\right)=d_{i}$, which implies the existence of integers $\mu_{i}, \nu_{i}$ satisfying

$$
a_{i}=\mu_{i} d_{i}, 1 \leq \mu_{i}<\frac{m_{i}}{d_{i}}, \quad \operatorname{gcd}\left(\mu_{i}, \frac{m_{i}}{d_{i}}\right)=1 ; \quad b_{i}=\nu_{i} d_{i}, 1 \leq \nu_{i}<\frac{m_{i}}{d_{i}}, \quad \operatorname{gcd}\left(\nu_{i}, \frac{m_{i}}{d_{i}}\right)=1 .
$$

Inserting $a_{i}$ and $b_{i}$ for $i=1, \ldots, r$ from (13) in (12) yields

$$
\nu_{1} d_{1} \equiv \lambda \mu_{1} d_{1} \quad \bmod m_{1}, \ldots, \nu_{r} d_{r} \equiv \lambda \mu_{r} d_{r} \quad \bmod m_{r} .
$$

We divide the i-th congruence by $d_{i}$ and multiply with $\kappa_{i}$, the multiplicative inverse of $\mu_{i}$ modulo $m_{i} / d_{i}$. Thus each congruence is solved for $\lambda$ and we arrive at the following system equivalent to (12).

$$
\lambda \equiv \kappa_{1} \nu_{1} \quad \bmod \frac{m_{1}}{d_{1}}, \ldots, \lambda \equiv \kappa_{r} \nu_{r} \quad \bmod \frac{m_{r}}{d_{r}}
$$

To prove the solvability of (14) by Lemma 5 we first notice that $\operatorname{gcd}\left(m_{i}, m_{j}\right) \leq 2$ for $i \neq j$ implies $\operatorname{gcd}\left(\left(m_{i} / d_{i}\right),\left(m_{j} / d_{j}\right)\right) \leq 2$ for $i, j=1, \ldots, r$. Suppose now that $m_{i} / d_{i}$ is even. As $\operatorname{gcd}\left(\mu_{i},\left(m_{i} / d_{i}\right)\right)=1$, see (13), $\mu_{i}$ must be odd. Also $\kappa_{i}$ is odd because of $\operatorname{gcd}\left(\kappa_{i},\left(m_{i} / d_{i}\right)\right)=1$. If for $i \neq j$ both $m_{i} / d_{i}$ and $m_{j} / d_{j}$ are even, then both $\kappa_{i} \nu_{i}$ and $\kappa_{j} \nu_{j}$ are odd, because all involved integers $\kappa_{i}, \nu_{i}, \kappa_{j}, \nu_{j}$ are odd. We conclude now by Lemma 5 that (14) is solvable, which finally confirms $b \in[a]$.

Lemma 6. Let $\Gamma=Z_{m_{1}} \otimes \cdots \otimes Z_{m_{r}}$ be isomorphic to $\Gamma^{\prime}=Z_{n_{1}} \otimes \cdots \otimes Z_{n_{s}}, \Gamma \simeq \Gamma^{\prime}$. Then $\Gamma$ is a gcd-group, if and only if $\Gamma^{\prime}$ is a gcd-group.

Proof. We may assume $m_{i} \geq 2$ for $i=1, \ldots, r$ and $n_{j} \geq 2$ for $j=1, \ldots, s$. For the following isomorphy and more basic facts about abelian groups we refer to Cohn [6].

$$
Z_{p q} \simeq Z_{p} \otimes Z_{q}, \text { if } \operatorname{gcd}(p, q)=1
$$


If the positive integer $m$ is written as a product of pairwise coprime prime powers, $m=$ $u_{1} \cdots u_{h}$, then

$$
Z_{m} \simeq Z_{u_{1}} \otimes \cdots \otimes Z_{u_{h}}
$$

We apply the decomposition (16) to every factor $Z_{m_{i}}, i=1, \ldots, r$, of $\Gamma$ and to every factor $Z_{n_{j}}, j=1, \ldots, s$, of $\Gamma^{\prime}$. So we obtain the "prime power representation" $\Gamma^{*}$, which is the same for $\Gamma$ and for $\Gamma^{\prime}$, if the factors are e. g. arranged in ascending order.

$$
\Gamma \simeq \Gamma^{*}=Z_{q_{1}} \otimes \cdots \otimes Z_{q_{t}} \simeq \Gamma^{\prime}, q_{j} \text { a prime power for } j=1, \ldots, t
$$

The following equivalences are easily checked.

$$
\begin{aligned}
& \operatorname{gcd}\left(m_{i}, m_{j}\right) \leq 2 \text { for every } i \neq j, i, j=1, \ldots, r \\
\Leftrightarrow \quad & \operatorname{gcd}\left(q_{k}, q_{l}\right) \leq 2 \text { for every } k \neq l, k, l=1, \ldots, t \\
\Leftrightarrow \quad & \operatorname{gcd}\left(n_{i}, n_{j}\right) \leq 2 \text { for every } i \neq j, i, j=1, \ldots, s
\end{aligned}
$$

Theorem 2 and (17) imply that $\Gamma$ is a gcd-group, if and only if $\Gamma^{*}$, respectively $\Gamma^{\prime}$, is a gcd-group.

Every finite abelian group $\tilde{\Gamma}$ can be represented as the direct product of cyclic groups.

$$
\tilde{\Gamma} \simeq Z_{m_{1}} \otimes \cdots \otimes Z_{m_{r}}=\Gamma
$$

We define $\tilde{\Gamma}$ to be a gcd-group, if $\Gamma$ is a gcd-group. Although the representation (18) may not be unique, this definition is correct by Lemma 6 .

Theorem 3. The finite abelian group $\Gamma$ is a gcd-group, if and only if $\Gamma$ is cyclic or $\Gamma$ is isomorphic to a group $\Gamma^{\prime}$ of the form

$$
\Gamma^{\prime}=Z_{2} \otimes \cdots \otimes Z_{2} \otimes Z_{n}, n \geq 2 .
$$

Proof. If $\Gamma$ is isomorphic to a group $\Gamma^{\prime}$ as stated in the theorem, then $\Gamma$ is a gcd-group by Theorem 2.

To prove the converse, let $\Gamma$ be a gcd-group. We may assume that $\Gamma$ is not cyclic. The prime power representation $\Gamma^{*}$ of $\Gamma$ is established as described in the proof of Lemma 6 . We start this representation with those orders which are a power of 2 , followed possibly by odd orders.

$$
\Gamma \simeq \Gamma^{*}=Z_{2} \otimes \cdots \otimes Z_{2} \otimes Z_{2^{\alpha}} \otimes Z_{u_{1}} \otimes \cdots \otimes Z_{u_{s}}, \quad \alpha \geq 1, u_{i} \text { odd for } i=1, \ldots, s
$$

Theorem 2 implies that there is at most one order $2^{\alpha}$ with $\alpha \geq 2$. Moreover, all odd orders $u_{1}, \ldots, u_{s}$ must be pairwise coprime. As $2^{\alpha}, u_{1}, \ldots, u_{s}$ are pairwise coprime integers, we deduce from (15) that

$$
Z_{2^{\alpha}} \otimes Z_{u_{1}} \otimes \cdots \otimes Z_{u_{s}} \simeq Z_{n} \text { for } n=2^{\alpha} u_{1} \cdots u_{s} .
$$

Now (19) implies

$$
\Gamma \simeq \Gamma^{\prime}=Z_{2} \otimes \cdots \otimes Z_{2} \otimes Z_{n}
$$




\section{$3 \quad$ Integral Cayley graphs over gcd-groups}

The following method to determine the eigenvectors and eigenvalues of Cayley graphs over abelian groups is due to Lovász [13], see also our description in [10]. We outline the main features of this method, which will be applied in this section.

The finite, additive, abelian group $\Gamma,|\Gamma|=n \geq 2$, is represented as the direct product of cyclic groups,

$$
\Gamma=Z_{m_{1}} \otimes \cdots \otimes Z_{m_{r}}, m_{i} \geq 2 \text { for } 1 \leq i \leq r .
$$

We consider the elements $x \in \Gamma$ as elements of the cartesian product $Z_{m_{1}} \times \cdots \times Z_{m_{r}}$,

$$
x=\left(x_{i}\right), x_{i} \in Z_{m_{i}}=\left\{0,1, \ldots, m_{i}-1\right\}, 1 \leq i \leq r .
$$

Addition is coordinatewise modulo $m_{i}$. A character $\psi$ of $\Gamma$ is a homomorphism from $\Gamma$ into the multiplicative group of complex $n$-th roots of unity. Denote by $e_{i}$ the unit vector with entry 1 in position $i$ and entry 0 in every position $j \neq i$. A character $\psi$ of $\Gamma$ is uniquely determined by its values $\psi\left(e_{i}\right), 1 \leq i \leq r$.

$$
x=\left(x_{i}\right)=\sum_{i=1}^{r} x_{i} e_{i}, \quad \psi(x)=\prod_{i=1}^{r}\left(\psi\left(e_{i}\right)\right)^{x_{i}}
$$

The value of $\psi\left(e_{i}\right)$ must be an $m_{i}$-th root of unity. There are $m_{i}$ possible choices for this value. Let $\zeta_{i}$ be a fixed primitive $m_{i}$-th root of unity for every $i, 1 \leq i \leq r$. For every $\alpha=\left(\alpha_{i}\right) \in \Gamma$ a character $\psi_{\alpha}$ can be uniquely defined by

$$
\psi_{\alpha}\left(e_{i}\right)=\zeta_{i}^{\alpha_{i}}, 1 \leq i \leq r .
$$

Combining (21) and (22) yields

$$
\psi_{\alpha}(x)=\prod_{i=1}^{r} \zeta_{i}^{\alpha_{i} x_{i}} \text { for } \alpha=\left(\alpha_{i}\right) \in \Gamma \text { and } x=\left(x_{i}\right) \in \Gamma .
$$

Thus all $|\Gamma|=m_{1} \cdots m_{r}=n$ characters of the abelian group $\Gamma$ can be obtained.

Lemma 7. Let $\psi_{0}, \ldots, \psi_{n-1}$ be the distinct characters of the additive abelian group $\Gamma=$ $\left\{w_{0}, \ldots, w_{n-1}\right\}, S \subseteq \Gamma, 0 \notin S,-S=S$. Assume that $A(G)=A=\left(a_{i, j}\right)$ is the adjacency matrix of $G=\operatorname{Cay}(\Gamma, S)$ with respect to the given ordering of the vertex set $V(G)=\Gamma$.

$$
a_{i, j}=\left\{\begin{array}{l}
1, \text { if } w_{i} \text { is adjacent to } w_{j} \\
0, \text { if } w_{i} \text { and } w_{j} \text { are not adjacent }
\end{array} \quad, 0 \leq i \leq n-1,0 \leq j \leq n-1\right.
$$

Then the vectors $\left(\psi_{i}\left(w_{j}\right)\right)_{j=0, \ldots, n-1}, 0 \leq i \leq n-1$, represent an orthogonal basis of $\mathbb{C}^{n}$ consisting of eigenvectors of $A$. To the eigenvector $\left(\psi_{i}\left(w_{j}\right)\right)_{j=0, \ldots, n-1}$ belongs the eigenvalue

$$
\psi_{i}(S)=\sum_{s \in S} \psi_{i}(s)
$$


There is a unique character $\psi_{w_{i}}$ associated with every $w_{i} \in \Gamma$ according to (23). So we may assume in Lemma 7 that $\psi_{i}=\psi_{w_{i}}$ for $i=0, \ldots, n-1$. Let us call the $n \times n$-matrix

$$
H(\Gamma)=\left(\psi_{w_{i}}\left(w_{j}\right)\right), 0 \leq i \leq n-1,0 \leq j \leq n-1,
$$

the character matrix of $\Gamma$ with respect to the given ordering of the elements of $\Gamma$. Here we always assume that $\Gamma$ is represented by (20) as a direct product of cyclic groups and that the elements of $\Gamma$ are ordered lexicographically increasing. Then $w_{0}$ is the zero element of $\Gamma$. Moreover, by (23) the character matrix $H(\Gamma)$ becomes the Kronecker product of the character matrices of the cyclic factors of $\Gamma$,

$$
\Gamma=Z_{m_{1}} \otimes \cdots \otimes Z_{m_{r}} \text { implies } H(\Gamma)=H\left(Z_{m_{1}}\right) \otimes \cdots \otimes H\left(Z_{m_{r}}\right) .
$$

We remind that the Kronecker product $A \otimes B$ of matrices $A$ and $B$ is defined by replacing the entry $a_{i, j}$ of $A$ by $a_{i, j} B$ for all $i, j$. For every Cayley graph $G=\operatorname{Cay}(\Gamma, S)$ the rows of $H(\Gamma)$ represent an orthogonal basis of $\mathbb{C}^{n}$ consisting of eigenvectors of $G$, respectively $A(G)$. The corresponding eigenvalues are obtained by $H(\Gamma) c_{S, \Gamma}$, the product of $H(\Gamma)$ and the characteristic (column) vector $c_{S, \Gamma}$ of $S$ in $\Gamma$,

$$
c_{S, \Gamma}(i)=\left\{\begin{array}{l}
1, \text { if } w_{i} \in S \\
0, \text { if } w_{i} \notin S
\end{array}, 0 \leq i \leq n-1 .\right.
$$

Consider the situation, when $\Gamma$ is a cyclic group, $\Gamma=Z_{n}, n \geq 2$. Let $\omega_{n}$ be a primitive $n$-th root of unity. Setting $r=1$ and $\zeta_{1}=\omega_{n}$ in (23) we establish the character matrix $H\left(Z_{n}\right)=F_{n}$ according to the natural ordering of the elements $0,1, \ldots, n-1$.

$$
F_{n}=\left(\left(\omega_{n}\right)^{i j}\right), 0 \leq i \leq n-1,0 \leq j \leq n-1
$$

Observe that all entries in the first row and in the first column of $F_{n}$ are equal to 1 . For a divisor $\delta$ of $n, 1 \leq \delta \leq n$, we simplify the notation of the characteristic vector of the gcd-set $S_{Z_{n}}(\delta)$ in $Z_{n}$ to $c_{\delta, n}$,

$$
c_{\delta, n}(i)=\left\{\begin{array}{l}
1, \text { if } \operatorname{gcd}(i, n)=\delta \\
0, \text { otherwise }
\end{array}, 0 \leq i \leq n-1 .\right.
$$

For $\delta<n$ we have $0 \notin S_{Z_{n}}(\delta)$. So the Cayley graph $\operatorname{Cay}\left(Z_{n}, S_{Z_{n}}(\delta)\right)$ is well defined. It is integral by Corollary 1 . The eigenvalues of this graph are the entries of $F_{n} c_{\delta, n}$. Therefore, this vector is integral, which is also trivially true for $\delta=n$,

$$
F_{n} c_{\delta, n} \in Z^{n} \text { for every positive divisor } \delta \text { of } n .
$$

The only quadratic primitive root is -1 . This implies that $H\left(Z_{2}\right)=F_{2}$ is the elementary Hadamard matrix (see [12])

$$
F_{2}=\left(\begin{array}{rr}
1 & 1 \\
1 & -1
\end{array}\right)
$$


By (24) the character matrix of the $r$-fold direct product $Z_{2} \otimes \cdots \otimes Z_{2}=Z_{2}^{r}$ is

$$
H\left(Z_{2}^{r}\right)=F_{2} \otimes \cdots \otimes F_{2}=F_{2}^{(r)},
$$

the $r$-fold Kronecker product of $F_{2}$ with itself, which is also a Hadamard matrix consisting of orthogonal rows with entries \pm 1 .

From now on let $\Gamma$ be a gcd-group. By Theorem 3 we may assume

$$
\Gamma=Z_{2}^{r} \otimes Z_{n}, r \geq 0, n \geq 2 .
$$

If we set $p=n-1$ and $q=2^{r}-1$, then we have $|\Gamma|-1=2^{r} n-1=q n+p$. We order the elements of $Z_{2}^{r}$, and $\Gamma$ lexicographically increasing.

$$
\begin{aligned}
& Z_{2}^{r}=\left\{a_{0}, a_{1}, \ldots, a_{q}\right\} \\
& a_{0}=(0, \ldots, 0,0), a_{1}=(0, \ldots, 0,1), \ldots, a_{q}=(1, \ldots, 1,1) \\
& \Gamma=\left\{w_{0}, w_{1}, \ldots, w_{q n+p}\right\}, \\
& w_{0}=\left(a_{0}, 0\right), w_{1}=\left(a_{0}, 1\right), \ldots, w_{p}=\left(a_{0}, p\right), \\
& \ldots \ldots \\
& w_{q n}=\left(a_{q}, 0\right), w_{q n+1}=\left(a_{q}, 1\right), \ldots, w_{q n+p}=\left(a_{q}, p\right) .
\end{aligned}
$$

The character matrix $H(\Gamma)$ with respect to the given ordering of elements becomes the Kronecker product of the character matrix $F_{2}^{(r)}$ of $Z_{2}^{r}$ and the character matrix $F_{n}$ of $Z_{n}$,

\begin{tabular}{|c|c|c|c|c|c|}
\hline & $\left(a_{0}, 0\right) \cdots\left(a_{0}, p\right)$ & $\cdots$ & $\left(a_{l}, 0\right) \cdots\left(a_{l}, p\right)$ & $\cdots$ & $\left(a_{q}, 0\right) \cdots\left(a_{q}, p\right)$ \\
\hline $\begin{array}{c}\left(a_{0}, 0\right) \\
\ldots \\
\left(a_{0}, p\right) \\
\end{array}$ & $\epsilon(0,0) F_{n}$ & $\begin{array}{l}\ldots \\
\ldots \\
\ldots \\
\end{array}$ & $\epsilon(0, l) F_{n}$ & $\begin{array}{l}\ldots \\
\cdots \\
\ldots \\
\end{array}$ & $\epsilon(0, q) F_{n}$ \\
\hline$\cdots$ & $\cdots$ & $\cdots$ & $\cdots$ & $\cdots$ & $\cdots$ \\
\hline $\begin{array}{c}\left(a_{j}, 0\right) \\
\ldots \\
\left(a_{j}, p\right)\end{array}$ & $\epsilon(j, 0) F_{n}$ & $\begin{array}{l}\cdots \\
\cdots \\
\cdots\end{array}$ & $\epsilon(j, l) F_{n}$ & $\begin{array}{l}\cdots \\
\ldots \\
\ldots\end{array}$ & $\epsilon(j, q) F_{n}$ \\
\hline$\cdots$ & $\cdots$ & $\cdots$ & $\cdots$ & $\cdots$ & $\cdots$ \\
\hline $\begin{array}{c}\left(a_{q}, 0\right) \\
\ldots \\
\left(a_{q}, p\right)\end{array}$ & $\epsilon(q, 0) F_{n}$ & $\begin{array}{l}\cdots \\
\cdots \\
\cdots \\
\end{array}$ & $\epsilon(q, l) F_{n}$ & $\begin{array}{l}\cdots \\
\cdots \\
\cdots \\
\end{array}$ & $\epsilon(q, q) F_{n}$ \\
\hline
\end{tabular}

$$
H(\Gamma)=F_{2}^{(r)} \otimes F_{n}
$$

This means that $H(\Gamma)$ consists of disjoint submatrices $\pm F_{n}$, because $F_{2}^{(r)}$ has only entries \pm 1 . The structure of $H(\Gamma)$ is displayed in Figure 1. Rows and columns are labelled with the elements of $\Gamma$. Observe that a label $\alpha$ at a row stands for the unique character $\psi_{\alpha}$. The sign $\epsilon(j, l) \in\{1,-1\}$ of a submatrix $F_{n}$ is the entry of $F_{2}^{(r)}$ in position $(j, l), 0 \leq j \leq$ $q, 0 \leq l \leq q$.

Figure 1: The structure of $H\left(Z_{2}^{r} \otimes Z_{n}\right)$. 
Let $m=\left(m_{1}, \ldots, m_{r}, m_{r+1}\right), m_{1}=\ldots=m_{r}=2, m_{r+1}=n$. Suppose that $d=$ $\left(d_{1}, \ldots, d_{r+1}\right)$ is a tuple of positive divisors of $m_{1}, \ldots, m_{r+1}, d_{i} \in\{1,2\}$ for $i=1, \ldots, r$, $d_{r+1}=\delta$ divides $n$. If $x=\left(x_{1}, \ldots, x_{r+1}\right) \in \Gamma=Z_{2}^{r} \otimes Z_{n}$ and $\operatorname{gcd}(x, m)=d$, then $x_{1}, \ldots, x_{r}$ are uniquely determined,

$$
x_{i}=\left\{\begin{array}{l}
1, \text { if } d_{i}=1 \\
0, \text { if } d_{i}=2
\end{array} \text { for } i=1, \ldots, r .\right.
$$

This means that the divisor tuple $d$ of $m$ determines a unique element $a_{l} \in Z_{2}^{r}$ such that

$$
\begin{aligned}
S_{\Gamma}(d) & =\left\{\left(a_{l}, b\right): b \in Z_{n}, \operatorname{gcd}(b, n)=\delta\right\} \\
& =\left\{w_{i} \in \Gamma: i=l n+b, 0 \leq b \leq p=n-1, \operatorname{gcd}(b, n)=\delta\right\} .
\end{aligned}
$$

The characteristic vector $c_{d, \Gamma}$ of $S_{\Gamma}(d)$ in $\Gamma$ may have nonzero entries only for positions $i=l n+b, b \in Z_{n}$. Its restriction to these positions is $x_{\delta, n}$, the characteristic vector of $S_{Z_{n}}(\delta)$ in $Z_{n}$. The vector $H(\Gamma) c_{d, \Gamma}$ is composed of $2^{r}$ disjoint vectors $\pm F_{n} c_{\delta, n}$, which by (25) have only integral entries. So $H(\Gamma) c_{d, \Gamma}$ has also only integral entries,

$$
H(\Gamma) c_{d, \Gamma} \in Z^{|\Gamma|} \text { for every divisor tuple } d \text { of } m \text {. }
$$

For different divisor tuples $d^{(1)}, \ldots, d^{(k)}$ of $m$ the sets of positions of $c_{d^{(1)}, \Gamma}, \ldots, c_{d^{(k)}, \Gamma}$ with entries 1 are pairwise disjoint. Therefore, these vectors are linearly independent in the rational space $\mathbb{Q}^{|\Gamma|}$.

From now on we abbreviate $H(\Gamma)=H, H=\left(h_{\alpha, \beta}\right), 0 \leq \alpha \leq|\Gamma|-1,0 \leq \beta \leq|\Gamma|-1$. We continue to use the notation established for (27). By $\tilde{D}$ we denote the set of all positive divisor tuples of $m=(2, \ldots, 2, n)$. The transpose of a vector $v$ is $v^{T}$. It is easily verified that

$$
\mathcal{A}=\left\{v \in \mathbb{Q}^{|\Gamma|}: H v \in \mathbb{Q}^{|\Gamma|}\right\}
$$

is a subspace of the rational space $\mathbb{Q}^{\Gamma}$. By (28) we see that

$$
\mathcal{D}=\operatorname{span}\left\{c_{d, \Gamma}: d \in \tilde{D}\right\} \subseteq \mathcal{A} .
$$

As $\left\{c_{d, \Gamma}: d \in \tilde{D}\right\}$ is a basis of $\mathcal{D}$, we have $\operatorname{dim}(\mathcal{D})=|\tilde{D}|=2^{r} \tau(n)$, where $\tau(n)$ is the number of positive divisors of $n$. The next lemma will enable us to show $\mathcal{D}=\mathcal{A}$.

Lemma 8. Let the elements of $\Gamma=Z^{r} \otimes Z_{n}$ be ordered as in (27), $\Gamma=\left\{w_{0}, \ldots, w_{q n+p}\right\}$, $q=2^{r}-1, p=n-1$, and let the character matrix $H=\left(h_{\alpha, \beta}\right)$ of $\Gamma$ be established with respect to this ordering of the elements (Figure 1). Moreover, let $v=\left(v_{0}, \ldots, v_{q n+p}\right)^{T} \in \mathcal{A}$, $u=\left(u_{0}, \ldots, u_{q n+p}\right)^{T}=H v$. Then

$$
\operatorname{gcd}\left(w_{s}, m\right)=\operatorname{gcd}\left(w_{t}, m\right) \text { implies } u_{s}=u_{t} \text { for every } s, t \in\{0,1, \ldots, q n+p\} .
$$

Proof. Notice that $v \in \mathcal{A}$ and $u=H v$ implies that the entries of $v$ and $u$ are rationals. Suppose $\operatorname{gcd}\left(w_{s}, m\right)=\operatorname{gcd}\left(w_{t}, m\right)=d, d=\left(d_{1}, \ldots, d_{r+1}\right), d_{i} \in\{1,2\}$ for $i=1, \ldots, r$, 
$d_{r+1}=\delta$ a positive divisor of $n$. As explained earlier, d uniquely determines elements $a_{l} \in Z_{2}^{r}$ and $b_{1}, b_{2} \in Z_{n}$ such that

$$
w_{s}=\left(a_{l}, b_{1}\right), w_{t}=\left(a_{l}, b_{2}\right), s=l n+b_{1}, t=l n+b_{2}, \operatorname{gcd}\left(b_{1}, n\right)=\operatorname{gcd}\left(b_{2}, n\right)=\delta .
$$

Rows $s$ and $t$ of $H$ belong to the same row of submatrices $\epsilon(l, g) F_{n}, 0 \leq g \leq q$ in Figure 1. We remind that $F_{n}=\left(\omega_{n}^{i j}\right), \omega_{n}$ a primitive $n$-th root of unity, $0 \leq i \leq p, 0 \leq j \leq p$, $p=n-1$.

$$
\begin{gathered}
u_{s}=\sum_{k=0}^{q n+p} h_{s, k} v_{k}=\sum_{g=0}^{q} \sum_{f=0}^{p} h_{l n+b_{1}, g n+f} v_{g n+f} \\
u_{s}=\sum_{g=0}^{q} \epsilon(l, g) \sum_{f=0}^{p} \omega_{n}^{b_{1} f} v_{g n+f} .
\end{gathered}
$$

Similarly we deduce

$$
u_{t}=\sum_{g=0}^{q} \epsilon(l, g) \sum_{f=0}^{p} \omega_{n}^{b_{2} f} v_{g n+f} .
$$

Setting $\omega_{n}^{b_{1}}=x$ in $(31)$ shows that $\omega_{n}^{b_{1}}$ is a root of the rational polynomial

$$
\psi(x)=\sum_{g=0}^{q} \epsilon(l, g) \sum_{f=0}^{p} x^{f} v_{g n+f}-u_{s} .
$$

As $\operatorname{gcd}\left(b_{1}, n\right)=\delta$ by $(30)$, we know that $\omega_{n}^{b_{1}}$ is an $(n / \delta)=\delta^{\prime}$-th root of unity. The irreducible polynomial over the rationals for a $\delta^{\prime}$-th root of unity is the cyclotomic polynomial $\Phi_{\delta^{\prime}}$ (see [6]). Therefore, we have $\psi(x)=M(x) \Phi_{\delta^{\prime}}(x)$ with a rational polynomial $M(x)$. Now we see by $(30), \operatorname{gcd}\left(b_{2}, n\right)=\delta$, that $\omega_{n}^{b_{2}}$ is also a $\delta^{\prime}$-th root of unity. So $\omega_{n}^{b_{2}}$ is also a root of $\Phi_{\delta^{\prime}}(x)$ and consequently also of $\psi(x)$.

$$
\psi\left(\omega_{n}^{b_{2}}\right)=\sum_{g=0}^{q} \epsilon(l, g) \sum_{f=0}^{p} \omega_{n}^{b_{2} f} v_{g n+f}-u_{s}=0 .
$$

Finally, (32) implies $u_{s}=u_{t}$.

Corollary 2. Assume that the conditions of Lemma 8 are satisfied. Let $\tilde{D}$ be the set of all positive divisor tuples of $m=(2, \ldots, 2, n)$. For $d \in \tilde{D}$ denote by $c_{d, \Gamma}$ the characteristic vector of $S_{\Gamma}(d)=\{w \in \Gamma: \operatorname{gcd}(w, m)=d\}$ in $\Gamma, \mathcal{D}=\operatorname{span}\left\{c_{d, \Gamma}: d \in \tilde{D}\right\}$. Then we have

$$
u=H v \in \mathcal{D} \text { for every } v \in \mathcal{A} .
$$

Proof. Suppose $d \in \tilde{D}$. By Lemma 8 the vector $u=H v$ has the same entry $\lambda_{d}$ in every position $j, w_{j} \in S_{\Gamma}(d)$. The sets $S_{\Gamma}(d), d \in \tilde{D}$ induce a partition of the set of all possible positions $\{0,1, \ldots,|\Gamma|-1\}=Z_{|\Gamma|}$ into disjoint subsets.

$$
S_{|\Gamma|}=\bigcup_{d \in \tilde{D}}\left\{j \in Z_{|\Gamma|}: w_{j} \in S_{\Gamma}(d)\right\}
$$

THE ELECTRONIC JOURNAL OF COMBINATORICS 18 (2011), \#P94 
This implies

$$
u=\sum_{d \in \tilde{D}} \lambda_{d} c_{d, \Gamma} \in \mathcal{D}
$$

Lemma 9. With the notations introduced for Lemma 8 and its corollary we have $\mathcal{D}=\mathcal{A}$.

Proof. By (29) $\mathcal{D}$ is a subspace of the linear space $\mathcal{A} \subseteq \mathbb{Q}^{|\Gamma|}$. Consider the mapping $\Delta$ defined by $\Delta(v)=H v$ for $v \in \mathcal{A}$. Corollary 2 shows that $\Delta$ maps $\mathcal{A}$ in $\mathcal{D}$. As the rows of $H$ are pairwise orthogonal and nonzero, this matrix is regular. Therefore, $\Delta$ is bijective, $\operatorname{dim}(\mathcal{D})=\operatorname{dim}(\mathcal{A}), \mathcal{D}=\mathcal{A}$.

As before let $\tilde{D}$ be the set of all positive divisor tuples $d$ of $m=(2, \ldots, 2, n)$. Remember that $\left\{c_{d, \Gamma}: d \in \tilde{D}\right\}$ is a basis of $\mathcal{D}=\mathcal{A}, \operatorname{dim}(\mathcal{A})=|\tilde{D}|$.

Lemma 10. Let $\Gamma=Z_{2}^{r} \otimes Z_{n}, S \subseteq \Gamma, 0 \notin S,-S=S$. The Cayley graph $G=\operatorname{Cay}(\Gamma, S)$ is integral, if and only $S=\emptyset$ or if there are positive divisor tuples $d^{(1)}, \ldots, d^{(k)}$ of $m=$ $(2, \ldots, 2, n)$ such that $S=S_{\Gamma}(D)$ for $D=\left\{d^{(1)}, \ldots, d^{(k)}\right\}$.

Proof. For $S=S_{\Gamma}(D)$ the Cayley graph $G=\operatorname{Cay}(\Gamma, S)$ is a gcd-graph, which is integral by Corollary 1.

To prove the converse, we skip the trivial case of $G$ being edgeless and assume that $G$ is integral, $S \neq \emptyset$. Let $c_{S, \Gamma}$ be the characteristic vector of $S$ with respect to the same ordering of the elements of $\Gamma$ which we used to establish the character matrix $H=H(\Gamma)$, see Figure 1. By Lemma 7 the entries of $H c_{S, \Gamma}$ are the eigenvalues of $G$, which are integral. This means $c_{S, \Gamma} \in \mathcal{A}$. Lemma 9 implies that there are positive, distinct divisor tuples $d^{(1)}, \ldots, d^{(k)}$ of $m$ such that

$$
c_{S, \Gamma}=\lambda_{1} c_{d^{(1)}, \Gamma}+\cdots+\lambda_{k} c_{d^{(k)}, \Gamma}, \lambda_{j} \in \mathbb{Q}, \lambda_{j} \neq 0 \text { for } j=1, \ldots, k .
$$

All vectors $c_{d^{(1)}, \Gamma}, \ldots, c_{d^{(k)}, \Gamma}$ have only 0,1 -entries and their sets of positions with entries 1 are pairwise disjoint. As $c_{S, \Gamma}$ has also only 0,1 -entries, we must have $\lambda_{1}=\cdots=\lambda_{k}=1$. Then $S$ becomes the disjoint union

$$
S=S_{\Gamma}\left(d^{(1)}\right) \cup \cdots \cup S_{\Gamma}\left(d^{(k)}\right)=S_{\Gamma}(D) .
$$

Theorem 4. Let $\Gamma$ be a gcd-group, $S \subseteq \Gamma, 0 \notin S,-S=S$. The Cayley graph $G=\operatorname{Cay}(\Gamma, S)$ is integral, if and only if $S$ belongs to the Boolean algebra $B(\Gamma)$ generated by the subgroups of $\Gamma$.

Proof. In [10] we showed that $S \in B(\Gamma)$ implies that $G$ is integral.

To prove the converse, we assume $S \neq \emptyset$ and $G=\operatorname{Cay}(\Gamma, S)$ integral. By Theorem 3 we know that there is a group $\Gamma^{\prime}=Z_{2}^{r} \otimes Z_{n}$ and a group isomorphism $\varphi: \Gamma \rightarrow \Gamma^{\prime}$. If we set $S^{\prime}=\varphi(S)$ and $G^{\prime}=\operatorname{Cay}\left(\Gamma^{\prime}, S^{\prime}\right)$, then $\varphi$ becomes also a graph isomorphism $\varphi: G \rightarrow G^{\prime}$. Therefore, $G^{\prime}$ is integral and $S^{\prime}$ is a gcd-set of $\Gamma^{\prime}$ by Lemma $10, S^{\prime} \in B_{g c d}\left(\Gamma^{\prime}\right)=B\left(\Gamma^{\prime}\right)$. The group isomorphism $\varphi$ provides a bijection between the sets in $B\left(\Gamma^{\prime}\right)$ and in $B(\Gamma)$. So we conclude $S \in B(\Gamma)$. 
Example. We have shown that for a gcd-group $\Gamma$ the integral Cayley graphs over $\Gamma$ are exactly the gcd-graphs over $\Gamma$. For an arbitrary group $\Gamma$ the number of integral Cayley graphs over $\Gamma$ may be considerably larger than the number of gcd-graphs over $\Gamma$.

Let $p$ be a prime number, $p \geq 5$. We determine the number of nonisomorphic gcdgraphs over $\Gamma=Z_{p} \otimes Z_{p}$. There are three possible divisor tuples of $(p, p)$ for the construction of a gcd-graph over $\Gamma$ : $(1,1),(1, p),(p, 1)$. From these tuples we can form 8 sets of divisor tuples:

$$
\begin{aligned}
& D_{1}=\emptyset, D_{2}=\{(1,1)\}, D_{3}=\{(1, p)\}, D_{4}=\{(p, 1)\}, D_{5}=\{(1,1),(1, p)\}, \\
& D_{6}=\{(1,1),(p, 1)\}, D_{7}=\{(1, p),(p, 1)\}, D_{8}=\{(1,1),(1, p),(p, 1)\} .
\end{aligned}
$$

Obviously, $D_{3}$ and $D_{4}$ generate isomorphic gcd-graphs over $\Gamma$, so do $D_{5}$ and $D_{6}$. Therefore, we cancel $D_{4}$ and $D_{6}$. The cardinalities $\left|S_{\Gamma}\left(D_{i}\right)\right|$ for $i \in\{1,2,3,5,7,8\}=M$ are in ascending order:

$$
0, p-1,2(p-1),(p-1)^{2}, p(p-1), p^{2}-1 .
$$

These are the degrees of regularity of the corresponding gcd-graphs $\operatorname{Cay}\left(\Gamma, S_{\Gamma}\left(D_{i}\right)\right), i \in$ $M$. As the above degree sequence is strictly increasing for $p \geq 5$, there are exactly 6 nonisomorphic gcd-graphs over $\Gamma=Z_{p} \otimes Z_{p}$.

Every element of $\Gamma=Z_{p} \otimes Z_{p}$ has order $p$ except for the zero element $(0,0)$. Denote by $[a]$ the cyclic subgroup generated by $a$. There are nonzero elements $a_{1}, \ldots, a_{p+1}$ in $\Gamma$ such that

$$
\Gamma=U_{1} \cup \cdots \cup U_{p+1}, U_{i}=\left[a_{i}\right], U_{i} \cap U_{j}=\{(0,0)\} \text { for } i \neq j .
$$

The sets

$$
S_{0}=\emptyset, S_{i}=\left(U_{1} \cup \cdots \cup U_{i}\right) \backslash\{(0,0)\}, 1 \leq i \leq p+1,
$$

belong to the Boolean algebra $B(\Gamma)$. Therefore, the Cayley graphs $G_{i}=\operatorname{Cay}\left(\Gamma, S_{i}\right), 0 \leq$ $i \leq p+1$, are integral. They are nonisomorphic, because they have pairwise distinct degrees of regularity: degree $\left(G_{i}\right)=i(p-1), 0 \leq i \leq p+1$. As there are exactly 6 nonisomorphic gcd-graphs over $\Gamma$, we conclude that there are at least $(p+2)-6=p-4$ nonisomorphic integral Cayley graphs over $\Gamma$, which are not gcd-graphs. An interesting task would be to determine for every prime number $p$ the number of all nonisomorphic integral Cayley graphs over $\Gamma=Z_{p} \otimes Z_{p}$.

\section{References}

[1] Abdollahi, A., and Vatandoost, E. Which Cayley graphs are integral? Electronic J. Comb. 16(1) (2009), R122, 1-17.

[2] Ahmadi, O., Alon, N., Blake, L. F., and Shparlinski, I. E. Graphs with integral spectrum. Linear Alg. Appl. 430 (2009), 547-552.

[3] Balinska, K., Cvetković, D., Rodosavljević, Z., Simić, S., and Stevanović, D. A survey on integral graphs. Univ. Beograd, Publ. Elektrotehn. Fak. Ser. Mat 13 (2003), 42-65. 
[4] Basić, M., Petković, M., And Stevanović, D. Perfect state transfer in integral circulant graphs. Appl. Math. Letters 22 (2009), 1117-1121.

[5] Biggs, N. Algebraic graph theory. Second Edition. Cambridge Mathematical Library. Cambridge University Press, 1993.

[6] Cohn, P. M. Basic Algebra. Springer, London, 2003.

[7] Godsil, C., And Royle, G. Algebraic graph theory. Graduate Texts in Mathematics. Vol 207. Springer, 2001.

[8] Harary, F., And Schwenk, A. J. Which graphs have integral spectra? Lecture Notes in Mathematics 406, Springer Verlag (1974), 45-50.

[9] Ilić, A. The energy of unitary cayley graphs. Linear Algebra and its Applications 431 (2009), 1881-1889.

[10] Klotz, W., And Sander, T. Integral Cayley graphs over abelian groups. Electronic J. Combinatorics 17 (2010), R81, 1-13.

[11] Klotz, W., And Sander, T. Some properties of unitary Cayley graphs. Electronic J. Combinatorics 14 (2007), R45, 1-12.

[12] van Lint, J. H., And Wilson, R. M. A course in combinatorics. Cambridge University Press, 1992.

[13] Lovász, L. Spectra of graphs with transitive groups. Priodica Mathematica Hungarica 6 (1975), 191-195.

[14] Rose H. E. A course in number theory. Oxford Science Publications. Oxford University Press, 1994.

[15] Saxena, N., Severini, S., and Shparlinski, I. Parameters of integral circulant graphs and periodic quantum dynamics. Intern. Journ. of Quantum Information 5 (2007), 417-430.

[16] So, W. Integral circulant graphs. Discrete Mathematics 306 (2005), 153-158. 